\title{
Detection of spreader nodes in Human-SARS-CoV protein- protein interaction network
}

\author{
Sovan Saha $^{\text {Corresp., } 1}$, Piyali Chatterjee ${ }^{2}$, Mita Nasipuri $^{3}$, Subhadip Basu $^{3}$ \\ ${ }^{1}$ Computer Science and Engineering, Institute of Engineering and Management, Kolkata, West Bengal, India \\ 2 Computer Science and Engineering, Netaji Subhash Engineering College, Kolkata, West Bengal, India \\ 3 Computer Science and Engineering, Jadavpur University, Kolkata, West Bengal, India \\ Corresponding Author: Sovan Saha \\ Email address: sovansaha12@gmail.com
}

The entire world is witnessing the coronavirus pandemic (COVID-19), caused by a novel coronavirus ( $\mathrm{n}-\mathrm{CoV}$ ) generally distinguished as Severe Acute Respiratory Syndrome Coronavirus 2 (SARS-CoV-2). SARS-CoV-2 promotes fatal chronic respiratory disease followed by multiple organ failure, ultimately putting an end to human life. International Committee on Taxonomy of Viruses(ICTV) has reached a consensus that SARS-CoV-2 is highly genetically similar (up to 89\%) to the Severe Acute Respiratory Syndrome Coronavirus (SARS-CoV), which had an outbreak in 2003. With this hypothesis, the current work focuses on identifying the spreader nodes in the SARS-CoV-human protein-protein interaction network (PPIN) to find possible lineage with the disease propagation pattern of the current pandemic. Various PPIN characteristics like edge ratio, neighborhood density, and node weight have been explored for defining a new feature spreadability index by which spreader proteins and protein-protein interaction (in the form of network edges) are identified. Top spreader nodes with a high spreadability index have been validated by Susceptible-Infected-Susceptible (SIS) disease model, first using a synthetic PPIN followed by a SARS-CoV-human PPIN. The ranked edges highlight the path of entire disease propagation from SARS-CoV to human PPIN (up to level-2 neighborhood). The developed network attribute, spreadability index, and the generated SIS model, compared with the other network centrality-based methodologies, perform better than the existing state-ofart. 
1 Detection of spreader nodes in Human-SARS-CoV 2 protein-protein interaction network

3

4

5

6

7

8

9

10

11

12

13

14

15

16

17

18

19

20

21

22

23

24

25

26

27

28

Sovan Saha ${ }^{1}$, Piyali Chatterjee ${ }^{2}$, Mita Nasipuri ${ }^{3}$, Subhadip Basu ${ }^{3}$

${ }^{1}$ Department of Computer Science \& Engineering, Institute of Engineering \& Management, Salt Lake Electronics Complex, Kolkata 700091, West Bengal, India.

${ }^{2}$ Department of Computer Science \& Engineering, Netaji Subhash Engineering College, Techno City, Panchpota, Kolkata 700152, West Bengal, India.

${ }^{3}$ Department of Computer Science \& Engineering, Jadavpur University, 188, Raja S.C. Mallick Road, Kolkata 700032, West Bengal, India.

Corresponding Author:

Sovan Saha ${ }^{1}$

Sector-V, Salt Lake Electronics Complex Kolkata-700091, West Bengal, India.

Email address: sovansaha12@gmail.com,sovan.saha@iemcal.com 


\section{Abstract}

The entire world is witnessing the coronavirus pandemic (COVID-19), caused by a novel coronavirus ( $\mathrm{n}-\mathrm{CoV}$ ) generally distinguished as Severe Acute Respiratory Syndrome Coronavirus 2 (SARS-CoV-2). SARS-CoV-2 promotes fatal chronic respiratory disease followed by multiple organ failure, ultimately putting an end to human life. International Committee on Taxonomy of Viruses (ICTV) has reached a consensus that SARS-CoV-2 is highly genetically similar (up to $89 \%$ ) to the Severe Acute Respiratory Syndrome Coronavirus (SARS-CoV), which had an outbreak in 2003. With this hypothesis, the current work focuses on identifying the spreader nodes in the SARS-CoV-human protein-protein interaction network (PPIN) to find possible lineage with the disease propagation pattern of the current pandemic. Various PPIN characteristics like edge ratio, neighborhood density, and node weight have been explored for defining a new feature spreadability index by which spreader proteins and protein-protein interaction (in the form of network edges) are identified. Top spreader nodes with a high spreadability index have been validated by Susceptible-Infected-Susceptible (SIS) disease model, first using a synthetic PPIN followed by a SARS-CoV-human PPIN. The ranked edges highlight the path of entire disease propagation from SARS-CoV to human PPIN (up to level-2 neighborhood). The developed network attribute, spreadability index, and the generated SIS model, compared with the other network centrality-based methodologies, perform better than the existing state-of-art.

\section{Introduction}

The COVID-19 pandemic registered its first case on 31 December 2019. First, it laid its foundation in the Chinese city of Wuhan (Hubei province) (Wang et al. 2020). Soon, it made several countries worldwide its victim by community spreading which ultimately compelled the World Health Organization (WHO) to declare a global health emergency on 30 January 2020 for the massive outbreak of COVID-19. Owing to its expected fatality rate, which is about $4 \%$, as projected by WHO, researchers from nations all over the world have joined their hands to work together to understand the spreading mechanisms of this virus SARS-CoV-2 (Heymann 2020; Huang et al. 2020; Liu \& Wang 2020; Zhou et al. 2020) and to find out all possible ways to save human lives from the dark shadow of COVID-19.

Coronavirus belongs to the family Coronaviridae. This single-stranded RNA virus affects not only humans but also mammals and birds too. Due to coronavirus, common fever/flu symptoms are noted in humans, followed by acute respiratory infections. Nevertheless, coronaviruses like Middle East Respiratory Syndrome (MERS) and Severe Acute Respiratory Syndrome (SARS) can create a global pandemic due to their infectious nature. Both of these coronaviruses are the member of genus Betacoronavirus under Coronaviridae. SARS started a significant outbreak in 2003, originating from Southern China. Seven hundred seventy-four deaths were reported among 8098 globally registered cases resulting in an estimated fatality rate of $14 \%-15 \%$. While MERS commenced in Saudi Arabia, creating an endemic in 2012. The world witnessed 858 deaths among 2494 registered positive cases. It generated a high fatality rate of $34.4 \%$ in comparison to SARS. 
SARS-CoV-2 is under the same Betacoronavirus genus as that of MERS and SARS coronavirus (Lu et al. 2020). It comprises several structural and non-structural proteins. The structural proteins include the envelope $(\mathrm{E})$ protein, membrane $(\mathrm{M})$ protein, nucleocapsid $(\mathrm{N})$ protein, and the spike (S) protein. Though SARS-CoV-2 has been identified recently, there is an intense scarcity of data and necessary information needed to gain immunity against SARS-CoV-2. Studies have revealed that SARS-CoV-2 is highly genetically similar to SARS-CoV based on several experimental genomic analyses (Hoffmann et al. 2020; Letko et al. 2020; Lu et al. 2020; Zhou et al. 2020). This is also the reason behind the naming of SARS-CoV-2 by the International Committee on Taxonomy of Viruses (ICTV). Due to this genetic similarity, the immunological study of SARS$\mathrm{CoV}$ may lead to the discovery of SARS-CoV-2 potential drug development.

A Protein-Protein Interaction Network (PPIN) has been used as the central component in identifying spreader nodes in SARS-CoV in the proposed methodology. PPIN is a very effective module for protein function determination (Cai et al. 2020; Hakala et al. 2020; Saha et al. 2019a; Saha et al. 2018; Saha et al. 2019b; Zhao et al. 2020) as well as in the identification of central/essential spreader nodes in the PPIN (Anthonisse 1971; He et al. 2021; Jeong et al. 2001; Joy et al. 2005; Li et al. 2011; Liu et al. 2019; Wen et al. 2020; Wuchty \& Stadler 2003; Zhong et al. 2021). The compactness of the PPIN and its transmission capability is estimated using centrality analysis. Anthonisse et al. (Anthonisse 1971) proposed a new centrality measure named Betweenness Centrality (BC). Another centrality measure, called closeness centrality (CC), is defined by Sabidussi et al. (Sabidussi 1966). Two other essential centrality measures: Degree centrality (DC) (Jeong et al. 2001) and Local average centrality (LAC) (Li et al. 2011), are also found to be very effective in this area of research.

Due to the high morbidity and mortality of SARS-CoV2, it has been felt that there is a pressing need to properly understand the way of viral infection transmission from SARS-CoV-2 PPIN to human PPIN. This paper considers SARS-CoV PPIN for this research study due to its high genetic similarity with SARS-CoV-2. Another primary motivation is to study the spreadability pattern of the ancestral strain of nCoV. In the proposed methodology, at first, SARS-CoV-Human PPIN (up to level-2) is formed from the collected datasets (Agrawal et al. 2017; Pfefferle et al. 2011). Once created, the spreader nodes are first identified in the SARS-CoV PPIN. Then its level-1 and level-2 interactors in the human PPIN are extracted using a new network attribute, i.e., spreadability index, which is a combination of three different network features: 1) edge ratio (Samadi \& Bouyer 2019) 2) neighborhood density (Samadi \& Bouyer 2019) and 3) node weight (Wang \& Wu 2013). The detected spreader nodes in the human PPIN are validated by the Susceptible, Infected, and Susceptible (SIS) epidemic disease model (Bailey 1975). Then the edges connecting two spreader nodes are ranked based on the average spreadability index. Thus, the ranked edges highlight the path through which viral infection gets mediated from SARS-CoV to human PPIN (up to level-2). The entire methodology can be categorized into 3-steps for 1) identifying the spreader nodes in the SARS-CoV and human PPIN using spreadability index, 2) validation of spreader nodes by SIS model, and 3) ranking of the spreader edges. 
106 Developing the spreadability index for raking edges in a host-pathogen PPIN to analyse the host's

107 viral infection propagation path is the primary contribution of this work. Furthermore, considering 108 the current investigation on SARS-CoV and the notable similarity with its successor virus, we also 109 attempt to shed light on the propagation pattern of viral infection of SARS-CoV2 in human PPIN.

110 In the following, we first describe the theory and methods for different network properties used to 111 extract the PPIN characteristics. Then we describe the 3-step methodology. First, the methodology 112 has been described using a synthetic PPIN (generated by Cytoscape (Shannon et al. 2003)). Then, 113 in the experimental results section, we have employed the developed method on the human-SARS114 CoV PPIN to identify the SARS-CoV viral infection propagation path in the human PPIN. Finally, 115 in the discussion section, we attempt to relate our findings with the ancestral virus, i.e., SARS$116 \mathrm{CoV}$, with its successor, i.e., SARS-CoV2, to study the SARS-CoV2 disease propagation may 117 follow the pattern from SARS-CoV.

\section{Theory \& Notations}

The viral infection gets mediated from one part of the PPIN to another through spreader nodes and edges (Brito \& Pinney 2017). Generally, in disease-specific PPIN models, at least two entities are involved: pathogen/Bait and host/Prey (Saha et al. 2017). In this research work, SARS-CoV takes the role of the former while human the latter one. Viral proteins of SARS-CoV tend to target their corresponding interaction with human proteins, which target its next level of proteins. So, the establishment of interactions between SARS-CoV and human occurs through connected nodes and edges of PPIN. But mostly, these viral proteins try to interact more with the central/hub proteins rather than the other proteins (Brito \& Pinney 2017). Thus, proper identification of central nodes (i.e., spreader nodes) is required. It is also confirmed that the interaction is not possible without the edges connecting two spreader nodes. Thus, these connecting edges are called spreader edges. The proposed methodology involves a proper study and assessment of various existing established PPIN features followed by identifying spreader nodes, which the SIS model has also verified. Before going into the detailed study about the proposed work, various network-based terminologies which are used in this work are discussed below:

\section{Protein-Protein Interaction Network (PPIN):}

When one protein interacts with another protein, it forms a network-like structure known as PPIN. Generally, it is portrayed as a graph where proteins are represented as nodes, and their corresponding connecting edges represent their interactions. Mathematically, PPIN can be highlighted as a graph $G_{n v}$, which consists of a set of vertices $v$ (nodes) connected by edges $e$ (links). Thus, $G_{n v}=(v, e)$ (Saha et al. 2014; Saha et al. 2019a).

\section{Level-1 and Level-2 proteins:}


143 In a PPIN, level-1 proteins of a node are those proteins that are in direct connection with that node, 144 i.e., its immediate neighbors, whereas level-2 proteins are those proteins that are indirectly 145 connected with level-1 proteins of that node, i.e., its indirect neighbors (Saha et al. 2014; Saha et 146 al. 2019a).

\section{Graph Centrality:}

149

Graph centrality is one of the essential aspects for the identification of significant nodes in a PPIN. The centrality of a node defines how relevant the node is in a PPIN or how much a node is centrally located in a PPIN.

153

\section{Betweenness Centrality (BC):}

155

156

157

158

159

160

161

162

163

164

165

166

167

168

169

170

171

172

173

174

175

BC (Anthonisse 1971) is one of the ways of measuring a node's impact on the transmission of information between every pair of nodes in a graph, considering that this transmission is always executed over the shortest path between them. Mathematically, it is defined as:

$$
C_{B}^{(u)}=\sum_{s \neq u \neq t} \frac{\rho(s, u, t)}{\rho(s, t)}
$$

where $\rho(s, t)$ is the total number of shortest paths from node $s$ to node $t$, and $\rho(s, u, t)$ is the number of those paths that pass through $u$.

\section{Closeness centrality (CC):}

CC (Sabidussi 1966) is a procedure for detecting nodes that transmit information within a network efficiently. Nodes with high closeness centrality values are considered to have the shortest distance to all available nodes in the network. It can be mathematically expressed as:

$$
C_{C}^{(u)}=\frac{\left|N_{u}\right|-1}{\sum_{v \in V} \operatorname{dist}(u, v)}
$$

where $\left|N_{u}\right|$ denotes the number of neighbors of node $u$ and $\operatorname{dist}(u, v)$ is the distance of the shortest path from node $u$ to node $v$.

\section{Degree centrality (DC):}

DC (Jeong et al. 2001) is considered the simplest among the available centrality measures that only count the degree of a node, i.e., the number of directly connected neighbors. Nodes having a high degree are said to be the highly connected module of the network. It is defined as:

$$
C_{D}^{(u)}=\left|N_{u}\right|
$$


176 where $\left|N_{u}\right|$ denotes the number of neighbors of node $u$.

177

178

179

180

181

182

183

184

185

186

187

188

189

190

191

192

193

194

195

196

197

198

199

200

201

\section{Local average centrality (LAC):}

LAC (Li et al. 2011) of a node represents how close its neighborhood proteins are. It is defined to be the local metric to compute the essentiality of the node for transmission ability by considering its modular nature, the mathematical model of which is highlighted as:

$$
\operatorname{LAC}(u)=\frac{\sum_{w \in N_{u}} \operatorname{deg}_{C_{u}}^{w}}{\left|N_{u}\right|}
$$

where $C_{u}$ is the subgraph induced by $N_{u}$ (i.e., the number of neighbors of node $u$ ) and $\operatorname{deg}_{c}{ }_{c}^{w}$ is the total number of nodes that are directly connected in $C_{u}$.

\section{Ego Network}

Ego network of node $i\left(S_{i}\right)$ (Samadi \& Bouyer 2019) is defined as the grouping of node $i$ itself along with its corresponding level-1 neighbors and interconnections. N $\left(S_{i}\right)$ (Samadi \& Bouyer 2019) consists of the set of nodes which belong to the ego network, $S_{i}$ i.e. $\{i\} \cup \Gamma(i)$.

\section{Edge ratio}

The edge ratio of node $i$ (Samadi \& Bouyer 2019) is defined by the following equation:

$$
\text { Edge ratio }(i)=\frac{\left(\sum_{j \in(i)}\left|\Gamma(j)-N\left(S_{i}\right)\right|\right)+1}{\left(\frac{1}{2} \sum_{j \in \Gamma(j)}\left|\Gamma^{S_{i}}(J)\right|\right)+1}=\frac{E_{\text {out }}^{S_{i}}+1}{E_{\text {in }}^{S_{i}}+1}
$$

where $E_{\text {out }}^{S_{i}}$ is the total number of interactions between the ego network $S_{i}$ and the proteins outside it. $E_{i n}{ }_{i}$ is the total number of interactions among node $i$ 's neighbors. $\Gamma(i)$ denotes the level-1 neighbors of node $i . S_{i}$ is considered to be Ego network. $\Gamma^{S_{i}}(j)$ denotes node $j$ 's neighbors which belongs $S_{i}$. In the edge ratio, $E_{\text {out }}^{S_{i}}$ is positively related to the non-peripheral location of node $i$. A large number of interactions resulting from the ego network denotes that the node has a high level of interconnectivity between its neighbors. On the other hand, $E_{i n}{ }_{i}$ is negatively related to the intermodule location of node $i$. It represents the fact that the interconnectivity between neighbors is usually connected to the number of structural holes available around the node. Thus, when the neighbor's interconnectivity is low, the root or the central node $i$ gains more control of transmission flow among the neighbors. 
202

203

204

205

206

207

208

209

210

211

212

213

214

215

216

217

218

219

220

221

222

223

224

225

226

227

228

229

230

231

\section{Jaccard Dissimilarity}

The similarity between two nodes is determined by Jaccard dissimilarity (Jaccard 1912) based on their common neighbors. Jaccard dissimilarity of node $i$ and $j$ (dissimilarity $(i, j)$ ) is defined as:

$$
\operatorname{dissimilarity}(i, j)=1-\operatorname{sim}(i, j)=\frac{|\Gamma(i) \cap \Gamma(j)|}{|\Gamma(i) \cup \Gamma(j)|}
$$

where $|\Gamma(\mathrm{i}) \cap \Gamma(\mathrm{j})|$ refers to the number of common neighbors of $i$ and $j .|\Gamma(\mathrm{i}) \cup \Gamma(\mathrm{j})|$ is the total number of neighbors of $i$ and $j$. The similarity degree between $i$ and $j$ is considered more when they have more common neighbors. Whereas, when dissimilarity between the neighbors of a node is high, it guarantees that the only common node among the neighbors is the central node, which is termed a structural hole situation (Samadi \& Bouyer 2019).

\section{Neighborhood Diversity}

The neighborhood diversity (Samadi \& Bouyer 2019) is a significant parameter of a graph that is based on Jaccard dissimilarity. When the dissimilarity of the neighbors of a node is high, it assures that the central node is the only neighbor common among the neighbors of that node, i.e., it represents the structural hole situation. On the other hand, when a node's neighborhood diversity reaches its greatest value, it reveals that the neighbors have no other closer path. Hence, the neighbors should transmit or communicate through this node. Mathematically, it is defined as:

$$
\text { neighborhood_diversity }(i)=\sum_{j, k \in(i)} \operatorname{dissimilarity}(j, k)
$$

\section{Node weight}

Node weight (Wang \& Wu 2013) is a graph parameter used to assign weightage to a node in a graph. Node weight $w_{v}$ of node $v \in V$ in PPIN is interpreted as the average degree of all nodes in $G_{V}$, a sub-graph of a graph $G_{V}$. It is considered as another measure to determine the strength of connectivity of a node in a network. Mathematically, it is represented by

$$
w_{v}=\frac{\sum_{u \in V^{\prime \prime}} \operatorname{deg}(u)}{\left|V^{\prime \prime}\right|}
$$

where $V^{\prime \prime}$ is the set of nodes in $G_{V} \cdot\left|V^{\prime \prime}\right|$ is the number of nodes in $G_{V}$. And $\operatorname{deg}(u)$ is the degree of a node $u \in V^{\prime \prime}$.

\section{Dataset}

Three datasets are mainly used for the present study. They are 1) SARS-CoV PPIN (Pfefferle et al. 2011) which contains only interactions of viral SARS-CoV proteins. 2) SARS-CoV-Human PPIN (Pfefferle et al. 2011) contains interaction information of SARS-CoV and human proteins. 3) Human PPIN (Agrawal et al. 2017), which contains only interactions of human proteins. These 
232

233

234

235

236

237

238

239

240

241

242

243

244

245

246

247

248

249

250

251

252

253

254

255

256

257

258

259

260

261

262

263

264

265

266

267

datasets are mainly used to generate two types of PPIN: 1) Synthetic PPIN and 2) Biological PPIN. Synthetic PPINs are the randomly generated sample PPINs (nodes with edges) used for the detailed analysis and testing of the proposed methodology (for example, please see Figure 1). The algorithm of the same is discussed in the supplementary document. Biological PPINs are the complete PPINs generated from the above datasets on which the proposed methodology is executed after testing (for example, please the complete PPIN view of SARS-CoV and human PPIN added at the end of the Experimental Results and Discussion section).

\section{Methodology}

The proposed work can be mainly categorized into three sub-sections: 1) Identification of spreader nodes by spreadability index, 2) Validation of spreader nodes by SIS model, and 3) Ranking of spreader edges.

\section{Identification of spreader nodes by spreadability index}

The spreadability index of node $i$ is defined as the ability of node $i$ to mediate a viral infection in a PPIN. Mathematically it can be defined as:

$$
\begin{aligned}
& \text { Spreadability_index }(i)=(\text { Edge ratio }(i) \times \text { neighborhood_diversity }(i))+ \\
& \text { Node weight }\left(w_{i}\right)
\end{aligned}
$$

Nodes having a high spreadability index are termed as spreader nodes, i.e., if the viral proteins establish interactions with these nodes, then the viral infection can be mediated to a more significant number of nodes in a much short amount of time compared to the other nodes in PPIN.

Figure 1 represents a sample PPIN where each protein is denoted as a node while edges mark its interactions with other proteins. The PPIN consists of 33 nodes and 53 edges. The PPIN data and the protein names and interactions are given as input to the Cytoscape, which generates the network view as highlighted in Figure 1. Cytoscape is open-source software that is used for PPIN generation and visualization (Shannon et al. 2003). The spreadability index is computed on the synthetic PPIN, shown in Figure 1, using essential PPIN characteristics in this PPIN, as stated earlier. The same is compared to DC, BC, CC, and LAC, highlighted in Table 1 to Table 5.

In Figure 1, it can be observed that nodes 1, 24 are the essential spreaders. Node 1 connects the four densely connected modules of the PPIN, making this node the topper with the highest spreadability index. This node has been correctly ranked by all the methods except LAC and DC. Node 24, though, has a moderate edge ratio and node weight but is one of the most densely connected modules itself despite getting isolated from the main PPIN module of node 1. Moreover, node 24 has the highest neighborhood density. It establishes that the only path of transmission of information for nodes 26, 27, 25, 28, 29, 30, 31, 32, and 33 is node 24. Thus, if viral proteins of SARS-CoV establishes interaction with node 24 , then all the connected nodes will be indirectly coming under the interaction of viral proteins as the connected nodes have no interactions with other central nodes except node 24 . So, node 24 holds the second position for the spreadability 
268

269

270

271

272

273

274

275

276

277

278

279

280

281

282

283

284

285

286

287

288

289

290

291

292

293

294

295

296

297

298

299

300

301

302

303

304

305

index in our proposed methodology. Node 24 is not correctly identified as the second most influential spreader node by the other methods. Further assessment of the remaining nodes highlights the fact that the performance of the new attribute spreadability index in our proposed methodology is relatively better in comparison to the others.

\section{Validation of spreader nodes by SIS model}

To design the mathematical model for this infectious disease, the SIS Epidemic Model (Bailey 1975 ) is used in this proposed methodology by classifying the proteins in SARS-CoV-human PPIN based on their interactivity status (for more details, please see "Studied Models in epidemiology" section of the supplementary document). SIS refers to Susceptible, Infected and Susceptible states, which are generally considered the three probable protein states in a PPIN. 1) $\mathbf{S}$ - The susceptible states are the states of those human proteins with which viral proteins have not yet interacted, but they are at risk of getting interacted. In general, every protein in PPIN is initially in a susceptible state. 2) I - These infected states are the states of those human proteins with which viral proteins have interacted, and the viral infection gets mediated. 3) $\mathbf{S}$ - The susceptible states are the states of those human proteins that have lost their interaction with the viral proteins (due to antiviral therapies or change in interface residues (Brito \& Pinney 2017)) and again become susceptible. The interaction rate of the viral proteins with human proteins, the loss rate of interactivity of the human protein with the viral proteins (general assumption is that any protein after coming out of the infected state gets into a susceptible state again in one day), and the total number of proteins are usually provided as input to SIS model. If a protein gets into an infected state and has many neighbors, any neighbor can mediate viral infection. So, the final result is generated after 50 iterations for each protein in the infected state. The total number of proteins in the susceptible state after 50 iterations in the neighborhood of each protein in an infected state divided by the total number of proteins in the PPIN gives the interaction capability of the protein in an infected state. Thus, the spreader nodes identified by the spreadability index are validated by the interaction rate as generated by the SIS model for them. It can be observed from Table 1 to Table 5 that the proposed methodology has the highest SIS interaction rate of 2.46 with viral proteins (see Table 1) in comparison to others for their corresponding top 10 spreader nodes in the synthetic PPIN, as shown in Figure 1.

\section{Ranking of Spreader edges}

To show the ranking of interacting spreader edges, two synthetic PPINs: PPIN-1 and PPIN-2, have been considered in Figure 2. Node D, E, and F are the selected top spreader nodes in PPIN-1 by spreadability index, similarly explained with a synthetic PPIN in Figure 1. To avoid the complexity in the diagram, the top 5 nodes in PPIN-2 (see Table 1) are selected as spreader nodes. Red-colored edges are the interconnectivity within PPIN-1, while black-colored edges show the interconnectivity within PPIN-2. Green-colored spreader edges (i.e., edges connected with spreader nodes) show the interconnectivity between PPIN-1 and PPIN-2. Ranking of a spreader edge measures the interaction ability of a spreader edge with the viral proteins, i.e., how many

Peer) reviewing PDF | (2021:06:62539:1:1:NEW 24 Jul 2021) 
306

307

308

309

310

311

312

313

314

315

316

317

318

319

320

321

322

323

324

325

326

327

328

329

330

331

332

333

334

335

336

337

338

339

340

341

342

343

nodes get interacted with the viral proteins through that edge, and the viral infection gets mediated. Thus, all the spreading edges are ranked based on the average spreadability index of its connected spreader nodes. The ranked spreader edges in Figure 2 are highlighted in Table 6.

\section{Experimental Results \& Discussion}

The proposed methodology leads to the identification of spreader nodes and edges through a network characteristic, called spreader index which has also been checked and validated by the SIS model. Initially, the whole working module is implemented on synthetic PPINs, as shown in the Methodology section, and then on the SARS-CoV-human dataset. For this proposed methodology, three PPIN datasets have been curated, already stated in the dataset section. After removing self-loops and data redundancy, the final SARS-CoV PPIN consists of 17 interactions among 7 SARS-CoV unique proteins (proteins having only one frequency of occurrence). Only the densely interconnected SARS-CoV proteins having direct connections (level-1) with human proteins are considered rather than isolated proteins. SARS-CoV-Human PPIN includes 118 interactions between SARS-CoV and humans. It is used to fetch the level-1 interaction of human proteins for the corresponding SARS-CoV proteins in SARS-CoV PPIN. Human PPIN consists of 314384 interactions. It is utilized for getting the indirect interactions (level-2) of level-1 human proteins formed earlier. The application of the proposed methodology in SARS-CoV-human PPIN is highlighted in Figure 3. In Figure 3A, at first, SARS-CoV PPIN is displayed in which each protein is marked in red. After that, spreader nodes in SARS-CoV PPIN are identified by the spreadability index. They are denoted as blue nodes among the red. Once the spreader nodes are active (Figure 3B), the viral infection gets mediated through its corresponding direct partners, i.e., human-level-1 proteins (marked in deep green). Then, in Figure 3C, spreader nodes are identified in SARS-CoV level-1 human proteins (marked in yellow). The same will continue to SARS-CoV level-2 human proteins (light green nodes are the spreaders).

In Figure 4, SARS-CoV PPIN has been highlighted. There are mainly nine proteins, including E, M, ORF3A, ORF7A, S, N, ORF8A, ORF8AB, and ORF8B. The computed spreadability index of these proteins and the corresponding validation by the SIS model are highlighted in Table 7. It is also compared with other central/ influential spreader node detection methodologies like DC, CC, LAC, and BC, shown in Table 8, Table 9, Table 10, and Table 11. Similarly, spreader nodes are also identified in SARS-CoV's level-1 neighbors and level-2 neighbors (see Figure 5 and Figure $6)$.

The spreadability index plays a vital role in this proposed methodology. Spreader nodes are successfully identified by this scoring technique which covers all the aspects through which viral infection gets mediated from one node to another in a PPIN (Brito \& Pinney 2017). It should be mentioned here that while identifying spreader nodes in SARS-CoV level-2 human proteins, it has been noted that the number of nodes is getting increased significantly with the increment of successive levels. So, high, medium, and low thresholds (Zhang et al. 2016) have been applied, and the entire viral infection mediation through spreadability index is computationally assessed at 
344 each threshold. The network statistics of spreader nodes at each level of threshold are shown in 345 Table 12. It can be observed that threshold application is only implemented at SARS-CoV level-2 346 human proteins, not on others. This is because of the availability of a smaller number of nodes and 347 edges. Therefore, only nodes and edges having a shallow spreadability index have been discarded 348 at the first level.

349 Besides the identification of spreader nodes, spreader edges are also identified. The ranked edges 350 between SARS-CoV spreaders and its level-1 human spreaders are highlighted in Table 13. In 351 contrast, the ranked edges between SARS-CoV s level-1 and level-2 human spreaders at high, 352 medium, and low thresholds are highlighted in the supplementary Table S1, Table S2, and Table 353 S3, respectively. The supplementary document is available online here. The complete PPIN view 354 of SARS-CoV and human PPIN has been generated online (by using the pyvis module available 355 in python) under three circumstances:

356 1) All the nodes and edges are considered spreader nodes and edges respectively and ranked 357 accordingly.

358 https://yu2qkp7gwoinjwsebyw0xw-on.drv.tw/www.graph all.html/graph all.html

359 2) Selected Spreader nodes and edges are highlighted for the high threshold.

360 https://yu2qkp7gwoinjwsebyw0xw-on.drv.tw/www.high_threshold.com/graph_high_threshold.html

361 3) Selected Spreader nodes and edges are highlighted for the medium threshold.

https://yu2qkp7gwoinjwsebyw0xw-on.drv.tw/www.medium_threshold.com/graph_medium_threshold.html

4) Selected Spreader nodes and edges are highlighted for the low threshold.

https://yu2qkp7gwoinjwsebyw0xw-on.drv.tw/www.low threshold.com/graph low threshold.html

365 In the above-generated PPIN views, the blue, yellow, and green colors represent SARS-CoV 366 spreaders, level-1 human spreaders, and its level2 human spreaders. The remaining nodes are in 367 indigo.

368

369

370

371

372

373

374

375

376

377

\section{Conclusion}

The spreadability index is thus proved to be effective in detecting spreader nodes and edges in SARS-CoV-human PPIN and the cross-validation by the SIS model. Spreader nodes are the central nodes in the PPIN through which viral infection gets mediated to their successors. Simultaneously, if the spreader nodes are not connected with spreader edges, that would not have been possible. In a nutshell, it can be said that the proposed work exploits the possibility of understanding how viral infection gets mediated from the SARS-CoV PPIN to the human PPIN. It should be borne in mind that SARS-CoV2 is $\sim 89 \%$ genetically similar to its predecessor SARS-CoV (Chan et al. 2020). Therefore, it strongly reveals that the human proteins chosen as spreaders of SARS-CoV might be the potential targets of SARS-CoV2. So, the same concept of the Spreadability index is applied 
410

411

412

413

414

415

416

417

418

419

420

421

422

423

424

along with a unique fuzzy protein-protein interaction model to form SARS-CoV2-human PPIN in our other research work (Saha et al. 2020a). The formed PPIN is also compared (Saha et al. 2020b) with that of SARS-CoV2-Human PPIN generated in the work of Gordon et al. (Gordon et al. 2020). Henceforth, study and analysis of drug repurposing of COVID-19 are also implemented in the subsequent research work (Saha et al. 2020b). Thus, it explores a new direction in identifying essential drugs/vaccines for SARS-CoV2. Recently, the work is limited to only SARS$\mathrm{CoV} / \mathrm{SARS}-\mathrm{CoV} 2$, which can be further extended to other viral infectious diseases in our future work.

\section{Acknowledgements}

The authors received support (infrastructure facilities) from the "Center for Microprocessor Applications for Training Education and Research" laboratory of the Computer Science and Engineering Department, Jadavpur University, India. In addition, this project is partially supported by the Department of Biotechnology project (No. BT/PR16356/BID/7/596/2016), Ministry of Science and Technology, Government of India. There was no additional external funding received for this study.

\section{References}

BioSNAP: Network datasets: Human protein-protein interaction network. Available at https://snap.stanford.edu/biodata/datasets/10000/10000-PP-Pathways.html.

China releases genetic data on new coronavirus, now deadly | CIDRAP. Available at https://www.cidrap.umn.edu/news-perspective/2020/01/china-releases-genetic-data-new-coronavirus-nowdeadly.

Naming the coronavirus disease (COVID-19) and the virus that causes it. Available at https://www.who.int/emergencies/diseases/novel-coronavirus-2019/technical-guidance/naming-thecoronavirus-disease-(covid-2019)-and-the-virus-that-causes-it.

Statement on the meeting of the International Health Regulations (2005) Emergency Committee regarding the outbreak of novel coronavirus 2019 (n-CoV) on 23 January 2020. Available at https://www.who.int/newsroom/detail/23-01-2020-statement-on-the-meeting-of-the-international-health-regulations-(2005)emergency-committee-regarding-the-outbreak-of-novel-coronavirus-(2019-ncov).

Statement on the second meeting of the International Health Regulations (2005) Emergency Committee regarding the outbreak of novel coronavirus (2019-nCoV). Available at https://www.who.int/news-room/detail/30-012020-statement-on-the-second-meeting-of-the-international-health-regulations-(2005)-emergencycommittee-regarding-the-outbreak-of-novel-coronavirus-(2019-ncov).

WHO | Middle East respiratory syndrome coronavirus (MERS-CoV). Available at https://www.who.int/emergencies/mers-cov/en/.

WHO I Update 49 - SARS case fatality ratio, incubation period. Available at https://www.who.int/csr/sars/archive/2003_05_07a/en/.

World-Health-Organization Coronavirus disease (COVID-19) outbreak. Available at https://www.who.int/emergencies/diseases/novel-coronavirus-2019.

World Map | CDC. Available at https://www.cdc.gov/coronavirus/2019-ncov/cases-updates/worldmap.html?CDC_AA_refVal=https\%3A\%2F\%2Fwww.cdc.gov\%2Fcoronavirus\%2F2019ncov\%2Flocations-confirmed-cases.html.

Agrawal M, Zitnik M, and Leskovec J. 2017. Large-Scale Analysis of Disease Pathways in the Human Interactome. bioRxiv:189787-189787. 10.1101/189787

Anthonisse JM. 1971. The rush in a directed graph: Stichting Mathematisch Centrum.

Bailey NTJ. 1975. The mathematical theory of infectious diseases and its applications. 2nd edition. The mathematical theory of infectious diseases and its applications 2nd edition. 
425

426

427

428

429

430

431

432

433

434

435

436

437

438

439

440

441

442

443

444

445

446

447

448

449

450

451

452

453

454

455

456

457

458

459

460

461

462

463

464

465

466

467

468

469

470

471

472

473

474

475

476

477

478

479

Brito AF, and Pinney JW. 2017. Protein-Protein Interactions in Virus-Host Systems. Frontiers in Microbiology 8:1557.

Cai Y, Wang J, and Deng L. 2020. SDN2GO: An Integrated Deep Learning Model for Protein Function Prediction. Frontiers in Bioengineering and Biotechnology 8:391.

Chan JFW, Kok KH, Zhu Z, Chu H, To KKW, Yuan S, and Yuen KY. 2020. Genomic characterization of the 2019 novel human-pathogenic coronavirus isolated from a patient with atypical pneumonia after visiting Wuhan. Emerging Microbes and Infections 9:221-236. 10.1080/22221751.2020.1719902

Gordon CJ, Tchesnokov EP, Feng JY, Porter DP, and Götte M. 2020. The antiviral compound remdesivir potently inhibits RNAdependent RNA polymerase from Middle East respiratory syndrome coronavirus. American Society for Biochemistry and Molecular Biology Inc. p 4773-4779.

Hakala K, Kaewphan S, Bjorne J, Mehryary F, Moen H, Tolvanen M, Salakoski T, and Ginter F. 2020. Neural Network and Random Forest Models in Protein Function Prediction. IEEE/ACM Transactions on Computational Biology and Bioinformatics:1-1. 10.1109/TCBB.2020.3044230

He X, Kuang L, Chen Z, Tan Y, and Wang L. 2021. Method for Identifying Essential Proteins by Key Features of Proteins in a Novel Protein-Domain Network. Frontiers in Genetics 12:1081.

Heymann DL. 2020. Data sharing and outbreaks: best practice exemplified. Lancet Publishing Group. p 469-470.

Hoffmann M, Kleine-Weber H, Krüger N, Müller M, Drosten C, and Pöhlmann S. 2020. The novel coronavirus 2019 (2019-nCoV) uses the SARS-coronavirus receptor ACE2 and the cellular protease TMPRSS2 for entry into target cells. bioRxiv:2020.2001.2031.929042-922020.929001.929031.929042. 10.1101/2020.01.31.929042

Huang C, Wang Y, Li X, Ren L, Zhao J, Hu Y, Zhang L, Fan G, Xu J, Gu X, Cheng Z, Yu T, Xia J, Wei Y, Wu W, Xie X, Yin W, Li H, Liu M, Xiao Y, Gao H, Guo L, Xie J, Wang G, Jiang R, Gao Z, Jin Q, Wang J, and Cao B. 2020. Clinical features of patients infected with 2019 novel coronavirus in Wuhan, China. The Lancet 395:497-506. 10.1016/S0140-6736(20)30183-5

Jaccard P. 1912. THE DISTRIBUTION OF THE FLORA IN THE ALPINE ZONE. New Phytologist 11:37-50. 10.1111/j.1469-8137.1912.tb05611.x

Jeong H, Mason SP, Barabási AL, and Oltvai ZN. 2001. Lethality and centrality in protein networks. Nature 411:4142. $10.1038 / 35075138$

Joy MP, Brock A, Ingber DE, and Huang S. 2005. High-betweenness proteins in the yeast protein interaction network. Journal of Biomedicine and Biotechnology 2005:96-103. 10.1155/JBB.2005.96

Letko M, Marzi A, and Munster V. 2020. Functional assessment of cell entry and receptor usage for SARS-CoV-2 and other lineage B betacoronaviruses. Nature Microbiology 5:562-569. 10.1038/s41564-020-0688-y

Li M, Wang J, Chen X, Wang H, and Pan Y. 2011. A local average connectivity-based method for identifying essential proteins from the network level. Computational Biology and Chemistry 35:143-150. 10.1016/j.compbiolchem.2011.04.002

Liu W, Ma L, and Chen L. 2019. Identification of Essential Proteins by Using Complexes and Biological Information on Dynamic PPI Network. 2019 Seventh International Conference on Advanced Cloud and Big Data (CBD). p 127-132.

Liu X, and Wang X-J. 2020. Potential inhibitors for 2019-nCoV coronavirus M protease from clinically approved medicines. bioRxiv:2020.2001.2029.924100-922020.924101.924129.924100. 10.1101/2020.01.29.924100

Lu R, Zhao X, Li J, Niu P, Yang B, Wu H, Wang W, Song H, Huang B, Zhu N, Bi Y, Ma X, Zhan F, Wang L, Hu T, Zhou H, Hu Z, Zhou W, Zhao L, Chen J, Meng Y, Wang J, Lin Y, Yuan J, Xie Z, Ma J, Liu WJ, Wang D, Xu W, Holmes EC, Gao GF, Wu G, Chen W, Shi W, and Tan W. 2020. Genomic characterisation and epidemiology of 2019 novel coronavirus: implications for virus origins and receptor binding. The Lancet 395:565-574. 10.1016/S0140-6736(20)30251-8

Pfefferle S, Schöpf J, Kögl M, Friedel CC, Müller MA, Carbajo-Lozoya J, Stellberger T, von Dall'Armi E, Herzog P, Kallies S, Niemeyer D, Ditt V, Kuri T, Züst R, Pumpor K, Hilgenfeld R, Schwarz F, Zimmer R, Steffen I, Weber F, Thiel V, Herrler G, Thiel HJ, Schwegmann-Weßels C, Pöhlmann S, Haas J, Drosten C, and von Brunn A. 2011. The SARS-Coronavirus-host interactome: Identification of cyclophilins as target for panCoronavirus inhibitors. PLoS Pathogens 7. 10.1371/journal.ppat.1002331

Sabidussi G. 1966. The centrality index of a graph. Psychometrika 31:581-603. 10.1007/BF02289527

Saha S, Chatterjee P, Basu S, Kundu M, and Nasipuri M. 2014. FunPred-1: Protein function prediction from a protein interaction network using neighborhood analysis. Cellular and Molecular Biology Letters 19:675-691. 10.2478/s11658-014-0221-5

Saha S, Chatterjee P, Basu S, Nasipuri M, and Plewczynski D. 2019a. FunPred 3.0: improved protein function prediction using protein interaction network. PeerJ 7:e6830-e6830. 10.7717/peerj.6830

Peer) reviewing PDF | (2021:06:62539:1:1:NEW 24 Jul 2021) 
480

481

482

483

484

485

486

487

488

489

490

491

492

493

494

495

496

497

498

499

500

501

502

503

504

505

506

507

508

509

510

511

512

513

514

515

516

517

518

519

Saha S, Halder AK, Bandyopadhyay SS, Chatterjee P, Nasipuri M, and Basu S. 2020a. Computational modeling of Human-nCoV protein-protein interaction network. https://arxiv.org/abs/2005.04108

Saha S, Halder AK, Bandyopadhyay SS, Chatterjee P, Nasipuri M, Bose D, and Basu S. 2020b. Is Fostamatinib a possible drug for COVID-19? - A computational study. Open Science Framework. https://doi.org/10.31219/osf.io/7hgpj

Saha S, Prasad A, Chatterjee P, Basu S, and Nasipuri M. 2018. Protein function prediction from protein-protein interaction network using gene ontology based neighborhood analysis and physico-chemical features. Journal of Bioinformatics and Computational Biology 16:1850025-1850025. 10.1142/S0219720018500257

Saha S, Prasad A, Chatterjee P, Basu S, and Nasipuri M. 2019b. Protein function prediction from dynamic protein interaction network using gene expression data. Journal of Bioinformatics and Computational Biology. $10.1142 / \mathrm{S} 0219720019500252$

Saha S, Sengupta K, Chatterjee P, Basu S, and Nasipuri M. 2017. Analysis of protein targets in pathogen-host interaction in infectious diseases: a case study on Plasmodium falciparum and Homo sapiens interaction network. Briefings in Functional Genomics. 10.1093/bfgp/elx024

Samadi N, and Bouyer A. 2019. Identifying influential spreaders based on edge ratio and neighborhood diversity measures in complex networks. Computing 101:1147-1175. 10.1007/s00607-018-0659-9

Shannon P, Markiel A, Ozier O, Baliga NS, Wang JT, Ramage D, Amin N, Schwikowski B, and Ideker T. 2003. Cytoscape: a software environment for integrated models of biomolecular interaction networks. Genome Res 13:2498-2504. 10.1101/gr.1239303

Wang C, Horby PW, Hayden FG, and Gao GF. 2020. A novel coronavirus outbreak of global health concern. Lancet Publishing Group. p 470-473.

Wang S, and Wu F. 2013. Detecting overlapping protein complexes in PPI networks based on robustness. Proteome science 11:S18-S18. 10.1186/1477-5956-11-S1-S18

Wen C-G, Liu J-X, Qin L, Wang J, and Fang Y. 2020. Essential Proteins Identification Based on Integrated Network. In: Huang D-S, Bevilacqua V, and Hussain A, editors. Intelligent Computing Theories and Application. Cham: Springer International Publishing. p 81-91.

Wuchty S, and Stadler PF. 2003. Centers of complex networks. Journal of Theoretical Biology 223:45-53. 10.1016/S0022-5193(03)00071-7

Zhang Y, Lin H, Yang Z, Wang J, Liu Y, and Sang S. 2016. A method for predicting protein complex in dynamic PPI networks. BMC Bioinformatics 17:229-229. 10.1186/s12859-016-1101-y

Zhao B, Zhang Z, Jiang M, Hu S, Luo Y, and Wang L. 2020. NPF:network propagation for protein function prediction. BMC Bioinformatics 21:355. 10.1186/s12859-020-03663-7

Zhong J, Tang C, Peng W, Xie M, Sun Y, Tang Q, Xiao Q, and Yang J. 2021. A novel essential protein identification method based on PPI networks and gene expression data. BMC Bioinformatics 22:248. 10.1186/s12859-02104175-8

Zhou P, Yang XL, Wang XG, Hu B, Zhang L, Zhang W, Si HR, Zhu Y, Li B, Huang CL, Chen HD, Chen J, Luo Y, Guo H, Jiang RD, Liu MQ, Chen Y, Shen XR, Wang X, Zheng XS, Zhao K, Chen QJ, Deng F, Liu LL, Yan B, Zhan FX, Wang YY, Xiao GF, and Shi ZL. 2020. A pneumonia outbreak associated with a new coronavirus of probable bat origin. Nature 579:270-273. 10.1038/s41586-020-2012-7

PeerJ reviewing PDF | (2021:06:62539:1:1:NEW 24 Jul 2021) 


\section{Table 1 (on next page)}

Computation of spreadability index of synthetic Figure 1 and computation of spreadability rate of selected top 10 spreader nodes by SIS model. 


\begin{tabular}{|c|c|c|c|c|c|c|c|c|}
\hline Rank & Proteins & $E_{\text {out }}^{S_{i}}$ & $E_{i n}^{S_{i}}$ & $\begin{array}{l}\text { Edge } \\
\text { Ratio }\end{array}$ & $\begin{array}{c}\text { Neighborhood } \\
\text { Diversity }\end{array}$ & $\begin{array}{c}\text { Node } \\
\text { Weight }\end{array}$ & Spreadability Index & $\begin{array}{l}\text { Sum of SIS spreadability } \\
\text { rate of top } 10 \text { nodes }\end{array}$ \\
\hline 1 & 1 & 13 & 0 & 14.0 & 5.19 & 3.40 & 76.15 & \multirow{10}{*}{2.46} \\
\hline 2 & 24 & 4 & 2 & 1.66 & 12.5 & 1.87 & 22.70 & \\
\hline 3 & 4 & 6 & 1 & 3.50 & 3.63 & 2.40 & 15.11 & \\
\hline 4 & 5 & 8 & 3 & 2.25 & 4.39 & 3.60 & 13.48 & \\
\hline 5 & 19 & 6 & 2 & 2.33 & 3.8 & 2.80 & 11.66 & \\
\hline 6 & 23 & 5 & 4 & 1.20 & 6.58 & 3.00 & 10.89 & \\
\hline 7 & 17 & 4 & 1 & 2.50 & 3.33 & 2.00 & 10.33 & \\
\hline 8 & 6 & 7 & 0 & 8.00 & 0.87 & 3.00 & 10.00 & \\
\hline 9 & 2 & 4 & 4 & 1.00 & 6.84 & 2.83 & 9.68 & \\
\hline 10 & 22 & 6 & 4 & 1.40 & 3.88 & 3.60 & 9.03 & \\
\hline 11 & 25 & 7 & 0 & 8.00 & 0.71 & 3.00 & 8.71 & \multirow{23}{*}{--} \\
\hline 12 & 27 & 7 & 0 & 8.00 & 0.71 & 3.00 & 8.71 & \\
\hline 13 & 28 & 7 & 0 & 8.00 & 0.71 & 3.00 & 8.71 & \\
\hline 14 & 30 & 7 & 0 & 8.00 & 0.71 & 3.00 & 8.71 & \\
\hline 15 & 18 & 6 & 0 & 7.00 & 0.85 & 2.66 & 8.66 & \\
\hline 16 & 20 & 7 & 2 & 2.66 & 1.78 & 3.50 & 8.26 & \\
\hline 17 & 7 & 4 & 3 & 1.25 & 4.15 & 2.80 & 7.98 & \\
\hline 18 & 21 & 3 & 6 & 0.57 & 6.66 & 3.33 & 7.13 & \\
\hline 19 & 3 & 3 & 3 & 1.00 & 4.00 & 2.60 & 6.60 & \\
\hline 20 & 16 & 4 & 2 & 1.66 & 2.06 & 2.75 & 6.19 & \\
\hline 21 & 15 & 4 & 2 & 1.66 & 2.06 & 2.75 & 6.19 & \\
\hline 22 & 31 & 6 & 1 & 3.50 & 0.75 & 3.33 & 5.95 & \\
\hline 23 & 33 & 6 & 1 & 3.50 & 0.75 & 3.33 & 5.95 & \\
\hline 24 & 32 & 4 & 2 & 1.66 & 1.75 & 2.75 & 5.66 & \\
\hline 25 & 8 & 4 & 3 & 1.25 & 1.88 & 3.25 & 5.60 & \\
\hline 26 & 14 & 6 & 0 & 7.00 & 0.40 & 2.66 & 5.46 & \\
\hline 27 & 9 & 2 & 4 & 0.60 & 3.64 & 2.80 & 4.98 & \\
\hline 28 & 10 & 5 & 1 & 3.00 & 0.50 & 3.00 & 4.50 & \\
\hline 29 & 13 & 1 & 3 & 0.50 & 1.70 & 2.50 & 3.35 & \\
\hline 30 & 11 & 1 & 3 & 0.50 & 1.70 & 2.50 & 3.35 & \\
\hline 31 & 12 & 1 & 3 & 0.50 & 1.70 & 2.50 & 3.35 & \\
\hline 32 & 29 & 2 & 0 & 3.00 & 0.00 & 1.33 & 1.33 & \\
\hline 33 & 26 & 2 & 0 & 3.00 & 0.00 & 1.33 & 1.33 & \\
\hline
\end{tabular}




\section{Table 2 (on next page)}

Computation of CC of synthetic Figure 1 and computation of spreadability rate of selected top 10 spreader nodes by SIS model. 


\begin{tabular}{|c|c|c|c|}
\hline Rank & Proteins & Closeness Centrality & Sum of SIS spreadability rate of top 10 nodes \\
\hline 1 & 1 & 0.085 & \multirow{10}{*}{ 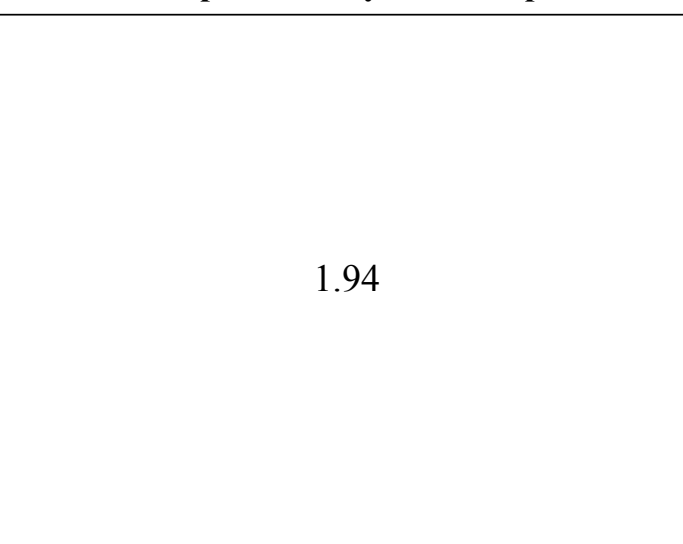 } \\
\hline 2 & 5 & 0.083 & \\
\hline 3 & 2 & 0.082 & \\
\hline 4 & 4 & 0.082 & \\
\hline 5 & 23 & 0.081 & \\
\hline 6 & 3 & 0.081 & \\
\hline 7 & 21 & 0.081 & \\
\hline 8 & 22 & 0.081 & \\
\hline 9 & 7 & 0.08 & \\
\hline 10 & 15 & 0.08 & \\
\hline 11 & 16 & 0.08 & \multirow{23}{*}{--} \\
\hline 12 & 19 & 0.079 & \\
\hline 13 & 14 & 0.079801 & \\
\hline 14 & 9 & 0.079602 & \\
\hline 15 & 20 & 0.079602 & \\
\hline 16 & 6 & 0.079602 & \\
\hline 17 & 8 & 0.079404 & \\
\hline 18 & 17 & 0.078818 & \\
\hline 19 & 10 & 0.078624 & \\
\hline 20 & 11 & 0.078049 & \\
\hline 21 & 12 & 0.078049 & \\
\hline 22 & 13 & 0.078049 & \\
\hline 23 & 18 & 0.07767 & \\
\hline 24 & 24 & 0.041558 & \\
\hline 25 & 32 & 0.041237 & \\
\hline 26 & 28 & 0.041237 & \\
\hline 27 & 30 & 0.041237 & \\
\hline 28 & 25 & 0.041237 & \\
\hline 29 & 27 & 0.041237 & \\
\hline 30 & 31 & 0.041184 & \\
\hline 31 & 33 & 0.041184 & \\
\hline 32 & 29 & 0.040921 & \\
\hline 33 & 26 & 0.040921 & \\
\hline
\end{tabular}




\section{Table 3(on next page)}

Computation of BC of synthetic Figure 1 and computation of spreadability rate of selected top 10 spreader nodes by SIS model. 


\begin{tabular}{|c|c|c|c|}
\hline Rank & Proteins & Betweeness Centrality & $\begin{array}{c}\text { Sum of SIS spreadability rate of top } 10 \\
\text { nodes }\end{array}$ \\
\hline 1 & 1 & 269.1 & \multirow{10}{*}{2.2} \\
\hline 2 & 2 & 117.93 & \\
\hline 3 & 4 & 117.1 & \\
\hline 4 & 3 & 114 & \\
\hline 5 & 5 & 108 & \\
\hline 6 & 24 & 57 & \\
\hline 7 & 23 & 56.4 & \\
\hline 8 & 19 & 45.56 & \\
\hline 9 & 17 & 39.1 & \\
\hline 10 & 7 & 36.9 & \\
\hline 11 & 6 & 32.9 & \multirow{23}{*}{--} \\
\hline 12 & 18 & 32 & \\
\hline 13 & 21 & 29.36 & \\
\hline 14 & 22 & 20.53 & \\
\hline 15 & 16 & 12.1 & \\
\hline 16 & 15 & 12.1 & \\
\hline 17 & 14 & 12.1 & \\
\hline 18 & 28 & 7 & \\
\hline 19 & 30 & 7 & \\
\hline 20 & 25 & 7 & \\
\hline 21 & 27 & 7 & \\
\hline 22 & 20 & 6.63 & \\
\hline 23 & 9 & 4.16 & \\
\hline 24 & 32 & 1 & \\
\hline 25 & 29 & 1 & \\
\hline 26 & 26 & 1 & \\
\hline 27 & 8 & 0 & \\
\hline 28 & 11 & 0 & \\
\hline 29 & 12 & 0 & \\
\hline 30 & 13 & 0 & \\
\hline 31 & 10 & 0 & \\
\hline 32 & 31 & 0 & \\
\hline 33 & 33 & 0 & \\
\hline
\end{tabular}


Table 4 (on next page)

Computation of LAC of synthetic Figure 1 and computation of spreadability rate of selected top 10 spreader nodes by SIS model. 


\begin{tabular}{|c|c|c|c|}
\hline Rank & Proteins & Local Average Centrality & $\begin{array}{l}\text { Sum of SIS Spreadability rate of top } \\
10 \text { nodes }\end{array}$ \\
\hline 1 & 21 & 2.4 & \multirow{10}{*}{2.19} \\
\hline 2 & 9 & 2 & \\
\hline 3 & 22 & 2 & \\
\hline 4 & 8 & 2 & \\
\hline 5 & 11 & 2 & \\
\hline 6 & 12 & 2 & \\
\hline 7 & 13 & 2 & \\
\hline 8 & 2 & 1.6 & \\
\hline 9 & 23 & 1.6 & \\
\hline 10 & 7 & 1.5 & \\
\hline 11 & 3 & 1.5 & \\
\hline 12 & 5 & 1.5 & \\
\hline 13 & 16 & 1.33 & \\
\hline 14 & 15 & 1.33 & \\
\hline 15 & 20 & 1.33 & \\
\hline 16 & 32 & 1.33 & \\
\hline 17 & 19 & 1 & \\
\hline 18 & 10 & 1 & \\
\hline 19 & 31 & 1 & \\
\hline 20 & 33 & 1 & \\
\hline 21 & 24 & 0.57 & \\
\hline 22 & 4 & 0.5 & \\
\hline 23 & 17 & 0.5 & \\
\hline 24 & 1 & 0 & \\
\hline 25 & 14 & 0 & \\
\hline 26 & 18 & 0 & \\
\hline 27 & 6 & 0 & -- \\
\hline 28 & 28 & 0 & \\
\hline 29 & 29 & 0 & \\
\hline 30 & 30 & 0 & \\
\hline 31 & 25 & 0 & \\
\hline 32 & 26 & 0 & \\
\hline 33 & 27 & 0 & \\
\hline
\end{tabular}




\section{Table 5 (on next page)}

Computation of DC of synthetic Figure 1 and computation of spreadability rate of selected top 10 spreader nodes by SIS model. 


\begin{tabular}{|c|c|c|c|}
\hline Rank & Proteins & Degree Centrality & $\begin{array}{c}\text { Sum of SIS spreadability rate of top } 10 \\
\text { nodes }\end{array}$ \\
\hline 1 & 24 & 7 & \multirow{10}{*}{2.3} \\
\hline 2 & 2 & 5 & \\
\hline 3 & 23 & 5 & \\
\hline 4 & 21 & 5 & \\
\hline 5 & 1 & 4 & \\
\hline 6 & 7 & 4 & \\
\hline 7 & 9 & 4 & \\
\hline 8 & 3 & 4 & \\
\hline 9 & 4 & 4 & \\
\hline 10 & 17 & 4 & \\
\hline 11 & 5 & 4 & \multirow{23}{*}{--} \\
\hline 12 & 22 & 4 & \\
\hline 13 & 19 & 4 & \\
\hline 14 & 8 & 3 & \\
\hline 15 & 11 & 3 & \\
\hline 16 & 12 & 3 & \\
\hline 17 & 13 & 3 & \\
\hline 18 & 16 & 3 & \\
\hline 19 & 15 & 3 & \\
\hline 20 & 20 & 3 & \\
\hline 21 & 32 & 3 & \\
\hline 22 & 10 & 2 & \\
\hline 23 & 14 & 2 & \\
\hline 24 & 18 & 2 & \\
\hline 25 & 6 & 2 & \\
\hline 26 & 28 & 2 & \\
\hline 27 & 29 & 2 & \\
\hline 28 & 30 & 2 & \\
\hline 29 & 25 & 2 & \\
\hline 30 & 26 & 2 & \\
\hline 31 & 27 & 2 & \\
\hline 32 & 31 & 2 & \\
\hline 33 & 33 & 2 & \\
\hline
\end{tabular}


Table 6(on next page)

Ranking of spreader edges for PPIN-1 and PPIN-2 in Figure 2. 


\begin{tabular}{|c|c|c|c|c|c|}
\hline & \multicolumn{2}{|c|}{ Spreader Edges } & & & \\
\hline Rank & $\begin{array}{c}\text { Spreader } \\
\text { nodes in } \\
\text { network 1 }\end{array}$ & $\begin{array}{c}\text { Spreader } \\
\text { nodes in } \\
\text { network 2 }\end{array}$ & $\begin{array}{c}\text { Spreadability Index } \\
\text { of spreader nodes in } \\
\text { network 1 }\end{array}$ & $\begin{array}{c}\text { Spreadability Index } \\
\text { of spreader nodes in } \\
\text { network 2 }\end{array}$ & $\begin{array}{c}\text { Ranking of } \\
\text { spreader edges }\end{array}$ \\
\hline 1 & F & 1 & 5.5 & 76.15 & 40.825 \\
\hline 2 & F & 24 & 5.5 & 22.70 & 14.104 \\
\hline 3 & D & 4 & 5.5 & 15.11 & 10.308 \\
\hline 4 & E & 5 & 4.7 & 13.48 & 9.0919 \\
\hline 5 & E & 19 & 4.7 & 11.66 & 8.1833 \\
\hline
\end{tabular}

1 


\section{Table 7 (on next page)}

Computation of spreadability index of SARS-CoV PPIN and computation of spreadability rate of selected top 6 spreader nodes by SIS model. 


\begin{tabular}{|c|c|c|c|c|c|c|c|c|c|}
\hline Rank & Proteins & $E_{\text {out }} \begin{array}{c}S_{i} \\
\text { on }\end{array}$ & $\underset{\text { in }}{\boldsymbol{S}_{\boldsymbol{i}}}$ & $\begin{array}{l}\text { Edge } \\
\text { Ratio }\end{array}$ & $\begin{array}{l}\text { Neighborhood } \\
\text { Density }\end{array}$ & $\begin{array}{c}\text { Node } \\
\text { Weight }\end{array}$ & $\begin{array}{l}\text { Spreadability } \\
\text { Index }\end{array}$ & $\begin{array}{c}\text { SIS } \\
\text { spreadability } \\
\text { rate of top } 6 \\
\text { nodes }\end{array}$ & $\begin{array}{c}\text { Sum of SIS } \\
\text { spreadability } \\
\text { rate of top } 6 \\
\text { nodes }\end{array}$ \\
\hline 1 & $\mathrm{M}$ & 7 & 3 & 2.0 & 3.845 & 3.4 & 11.090 & 1 & \multirow{6}{*}{2.935} \\
\hline 2 & $\mathrm{~S}$ & 6 & 3 & 1.75 & 4.047 & 3.2 & 10.283 & 0.2 & \\
\hline 3 & ORF8AB & 7 & 3 & 2.0 & 1.785 & 4.0 & 7.5714 & 1 & \\
\hline 4 & ORF8B & 5 & 5 & 1.0 & 3.464 & 3.8 & 7.2642 & 0.2 & \\
\hline 5 & $\mathrm{E}$ & 7 & 3 & 2.0 & 1.428 & 4.0 & 6.8571 & 0.25 & \\
\hline 6 & ORF3A & 2 & 8 & 0.333 & 9.249 & 3.428 & 6.5119 & 0.285 & \\
\hline 7 & ORF7A & 2 & 8 & 0.333 & 9.25 & 3.428 & 6.5119 & \multirow{3}{*}{--} & \multirow{3}{*}{--} \\
\hline 8 & ORF8A & 3 & 0 & 4.0 & 0.0 & 2.0 & 2 & & \\
\hline 9 & $\mathrm{~N}$ & 3 & 0 & 4.0 & 0.0 & 2.0 & 2 & & \\
\hline
\end{tabular}




\section{Table 8(on next page)}

Computation of degree centrality of SARS-CoV PPIN and computation of spreadability rate of selected top 6 spreader nodes by SIS model. 


\begin{tabular}{|c|c|c|c|c|}
\hline Rank & Proteins & Degree Centrality & $\begin{array}{c}\text { SIS spreadability rate } \\
\text { of top 6 nodes }\end{array}$ & $\begin{array}{c}\text { Sum of SIS spreadability } \\
\text { rate of top 6 nodes }\end{array}$ \\
\hline 1 & ORF7A & 6 & 0.285 & \multirow{2}{*}{1} \\
\hline 2 & ORF3A & 6 & 0.285 & \multirow{2}{*}{1.82} \\
\hline 3 & ORF8B & 4 & 0.2 & \\
\hline 4 & M & 4 & 0.6 & \\
\hline 5 & S & 4 & 0.2 & \\
\hline 6 & E & 3 & 0.25 & \\
\hline 7 & ORF8AB & 3 & -- & \\
\hline 8 & N & 1 & & \\
\hline 9 & ORF8A & 1 & & \\
\hline
\end{tabular}

1 


\section{Table 9 (on next page)}

Computation of closeness centrality of SARS-CoV PPIN and computation of spreadability rate of selected top 6 spreader nodes by SIS model. 


\begin{tabular}{|c|c|c|c|c|}
\hline Rank & Proteins & Closeness Centrality & $\begin{array}{l}\text { SIS spreadability rate of } \\
\text { top } 6 \text { nodes }\end{array}$ & $\begin{array}{c}\text { Sum of SIS } \\
\text { spreadability rate of } \\
\text { top } 6 \text { nodes }\end{array}$ \\
\hline 1 & ORF7A & 0.239 & 0.285 & \multirow{6}{*}{1.82} \\
\hline 2 & ORF3A & 0.239 & 0.285 & \\
\hline 3 & ORF8B & 0.224 & 0.2 & \\
\hline 4 & $\mathrm{M}$ & 0.224 & 0.6 & \\
\hline 5 & $\mathrm{~S}$ & 0.224 & 0.2 & \\
\hline 6 & $E$ & 0.215 & 0.25 & \\
\hline 7 & ORF8AB & 0.22 & \multirow{3}{*}{--} & \multirow{3}{*}{--} \\
\hline 8 & $\mathrm{~N}$ & 0.196 & & \\
\hline 9 & ORF8A & 0.196 & & \\
\hline
\end{tabular}

1 
Table $\mathbf{1 0}$ (on next page)

Computation of local average centrality of SARS-CoV PPIN and computation of spreadability rate of selected top 6 spreader nodes by SIS model. 


\begin{tabular}{|c|c|c|c|c|}
\hline Rank & Proteins & Local Average Centrality & $\begin{array}{l}\text { SIS spreadability rate } \\
\text { of top } 6 \text { nodes }\end{array}$ & $\begin{array}{c}\text { Sum of SIS } \\
\text { spreadability rate of } \\
\text { top } 6 \text { nodes }\end{array}$ \\
\hline 1 & ORF7A & 2.666 & 0.285 & \multirow{6}{*}{2.22} \\
\hline 2 & ORF3A & 2.666 & 0.285 & \\
\hline 3 & ORF8B & 2.5 & 0.2 & \\
\hline 4 & $\mathrm{E}$ & 2 & 0.25 & \\
\hline 5 & ORF8AB & 2 & 1 & \\
\hline 6 & $\mathrm{~S}$ & 1.5 & 0.2 & \\
\hline 7 & $\mathrm{M}$ & 1.5 & \multirow{3}{*}{--} & \multirow{3}{*}{--} \\
\hline 8 & $\mathrm{~N}$ & 0 & & \\
\hline 9 & ORF8A & 0 & & \\
\hline
\end{tabular}

1 


\section{Table 11 (on next page)}

Computation of betweeness centrality of SARS-CoV PPIN and computation of spreadability rate of selected top 6 spreader nodes by SIS model. 


\begin{tabular}{|c|c|c|c|c|}
\hline Rank & Proteins & Betweeness Centrality & SIS spreadability rate of top 6 nodes & $\begin{array}{c}\text { Sum of SIS } \\
\text { spreadability } \\
\text { rate of top } 6 \\
\text { nodes }\end{array}$ \\
\hline 1 & $M$ & 14 & 0.6 & \multirow{6}{*}{1.82} \\
\hline 2 & $\mathrm{~S}$ & 14 & 0.2 & \\
\hline 3 & ORF7A & 13.33 & 0.285 & \\
\hline 4 & ORF3A & 13.33 & 0.285 & \\
\hline 5 & ORF8B & 1.33 & 0.2 & \\
\hline 6 & $\mathrm{E}$ & 0 & 0.25 & \\
\hline 7 & ORF8AB & 0 & \multirow{3}{*}{--} & \multirow{3}{*}{--} \\
\hline 8 & $\mathrm{~N}$ & 0 & & \\
\hline 9 & ORF8A & 0 & & \\
\hline
\end{tabular}




\section{Table 12 (on next page)}

Network statistics of spreaders at three levels of thresholds. 


\begin{tabular}{|c|c|c|c|}
\hline Threshold & SARS-CoV spreaders & $\begin{array}{c}\text { SARS-CoV-s level 1 human } \\
\text { spreaders }\end{array}$ & $\begin{array}{c}\text { SARS-CoV-s level 2 } \\
\text { human spreaders }\end{array}$ \\
\hline High & 6 & 24 & 9 \\
\hline Medium & 6 & 24 & 22 \\
\hline Low & 6 & 24 & 111 \\
\hline
\end{tabular}

1 
Table $\mathbf{1 3}$ (on next page)

Ranked spreader edges between SARS-CoV spreaders and its level-1 human spreaders. 


\begin{tabular}{|c|c|c|c|}
\hline & \multicolumn{2}{|c|}{ Spreader Edges } & \multirow[b]{2}{*}{$\begin{array}{l}\text { Spreading ability of } \\
\text { spreader edges }\end{array}$} \\
\hline Rank & SARS-CoV Spreaders & $\begin{array}{c}\text { SARS-CoV s level } 1 \text { human } \\
\text { spreaders }\end{array}$ & \\
\hline 1 & $\mathrm{~N}$ & UBE2I & 679697.677 \\
\hline 2 & ORF3A & YWHAE & 500684.2755 \\
\hline 3 & ORF7A & SGTA & 428397.3206 \\
\hline 4 & ORF3A & PFDN5 & 273863.194 \\
\hline 5 & ORF3A & CAV1 & 264566.0653 \\
\hline 6 & $\mathrm{~N}$ & EEF1A1 & 241407.2776 \\
\hline 7 & ORF7A & VKORC1 & 187916.2768 \\
\hline 8 & $\mathrm{M}$ & IKBKB & 164728.3002 \\
\hline 9 & $\mathrm{~S}$ & NCL & 131643.7345 \\
\hline 10 & $\mathrm{~N}$ & PPIA & 125719.6427 \\
\hline 11 & $\mathrm{~S}$ & EIF3F & 119529.0273 \\
\hline 12 & ORF7A & BCL2 & 119299.092 \\
\hline 13 & ORF3A & DCTN2 & 92293.0019 \\
\hline 14 & $\mathrm{E}$ & BCL2L1 & 89404.47117 \\
\hline 15 & ORF7A & BCL2L1 & 89404.29855 \\
\hline 16 & ORF7A & MCL1 & 63953.80825 \\
\hline 17 & $\mathrm{~S}$ & CLEC4G & 27477.4133 \\
\hline 18 & ORF7A & BCL2L2 & 22974.97399 \\
\hline 19 & ORF7A & BCL2A1 & 22252.28441 \\
\hline 20 & $\mathrm{~S}$ & ACE2 & 18775.88601 \\
\hline 21 & $\mathrm{~S}$ & CEACAM1 & 14834.82402 \\
\hline 22 & $\mathrm{~S}$ & CD209 & 12215.99362 \\
\hline 23 & ORF7A & SMOC1 & 6068.990602 \\
\hline 24 & $\mathrm{~S}$ & CLEC4M & 3844.528751 \\
\hline 25 & $\mathrm{~S}$ & SFTPD & 119.09278 \\
\hline
\end{tabular}


Figure 1

Synthetic PPIN1.

The PPIN consists of 33 nodes and 53 edges. Nodes 1, 24 are the essential spreaders. Node 1 connects the four densely connected modules of the PPIN, which turns this node to stand in the first position having the highest spreadability index. Node 24 holds the second position for the spreadability index. Node 24 is one of the most densely connected modules itself despite getting isolated from the main PPIN module of node 1. 


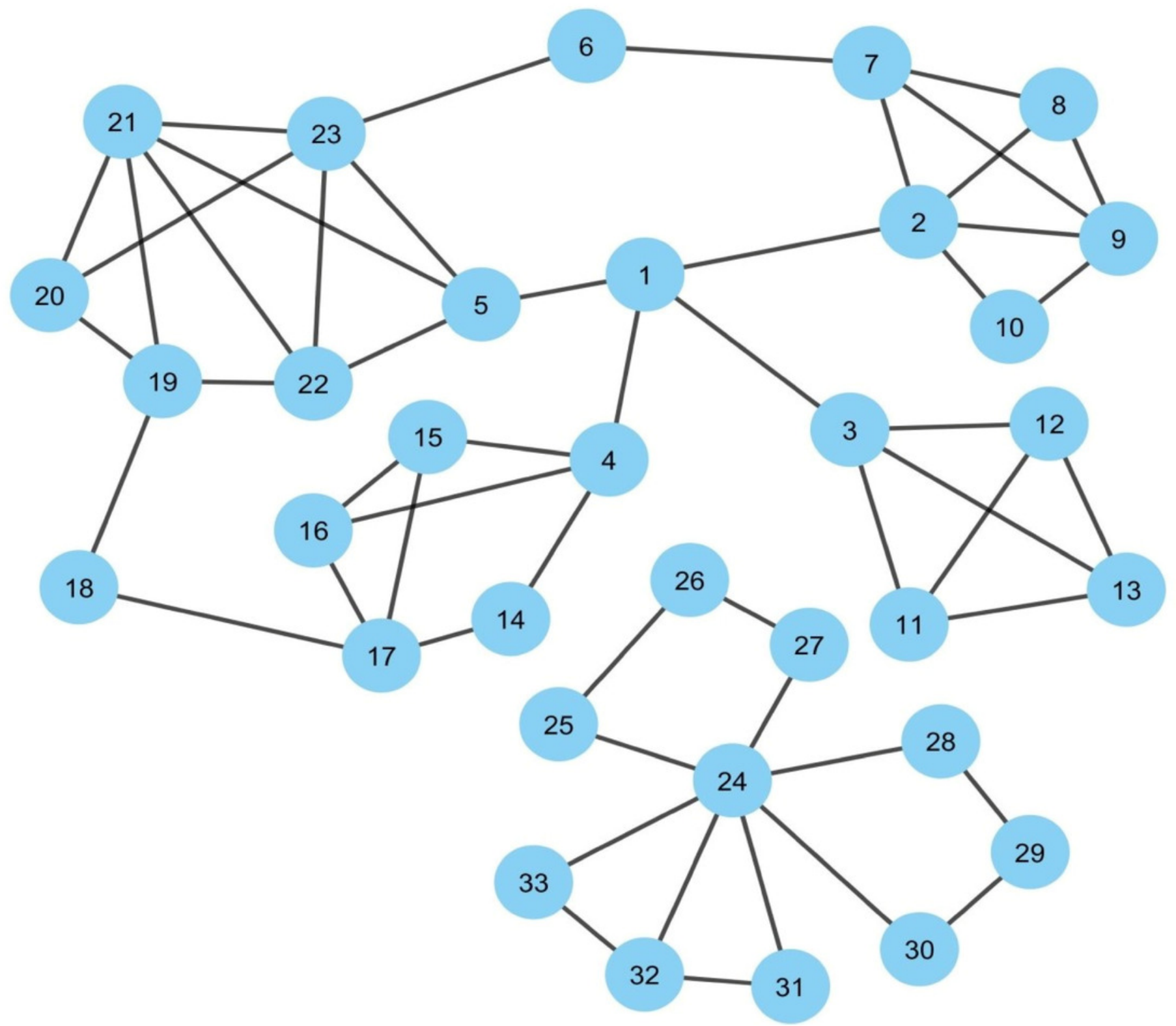


Figure 2

Ranking of Spreader edges.

Two synthetic PPINs: PPIN-1 and PPIN-2, have been considered for ranking spreader edges based on the spreadability index. Red-colored edges are the interconnectivity within PPIN-1, while black-colored edges show the interconnectivity within PPIN-2. Nodes D, E and F, are the detected spreader nodes of PPIN-1, whereas nodes 1, 4, 5, 19 and 24 are the detected spreader nodes of PPIN2. Green-colored spreader edges (i.e., edges connected with spreader nodes) show the interconnectivity between PPIN-1 and PPIN-2. The thickness of the edges varies with the order of ranking.

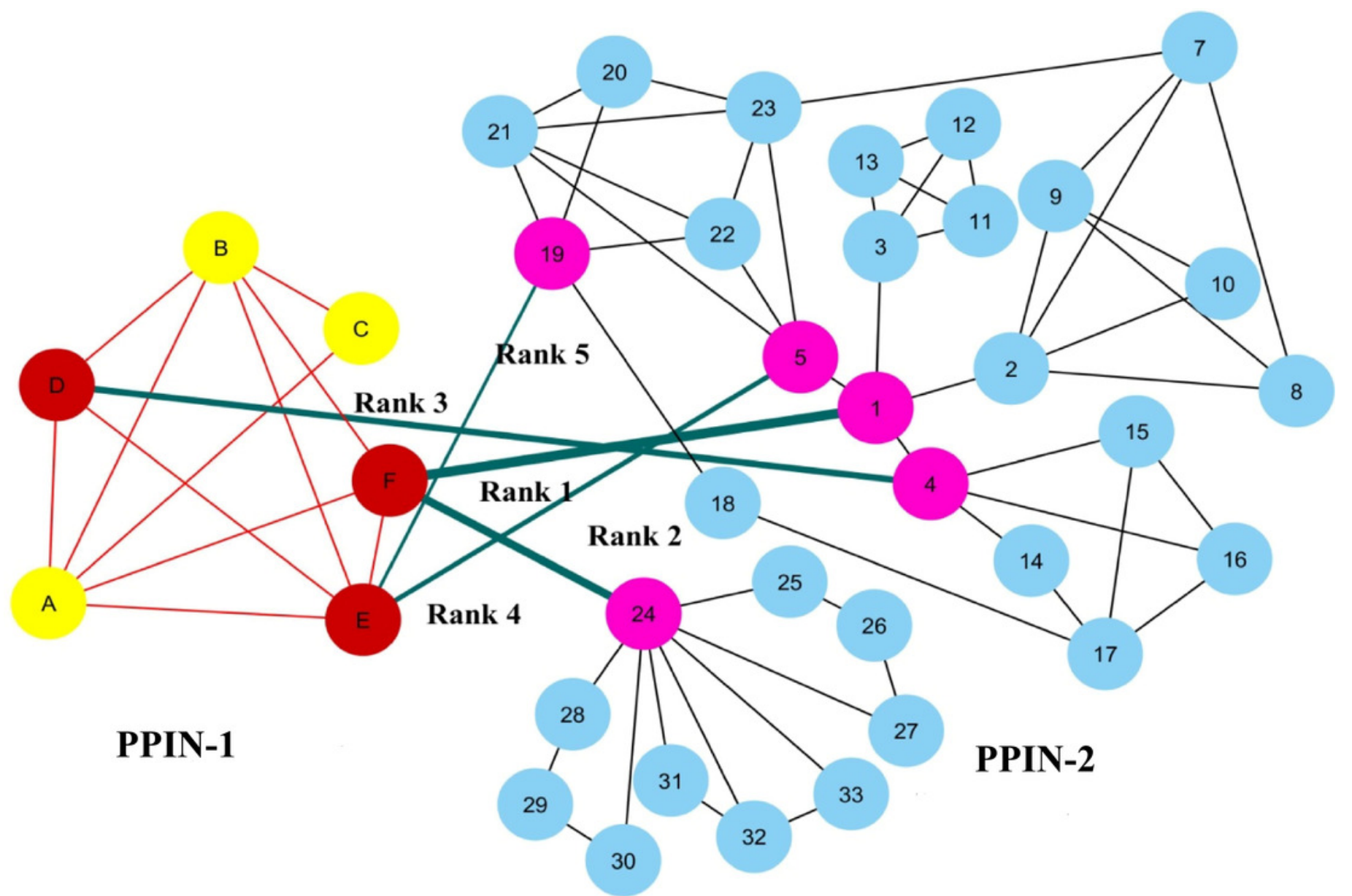


Figure 3

Mechanism of transmission of viral infection.

SARS-CoV spreaders establish interaction with human spreader proteins, and the viral infection gets mediated from SARS-CoV PPIN to human PPIN through them. A. PPIN of SARS$\mathrm{CoV}$ (red) in which spreader nodes are marked as blue. B. Interaction of SARS-CoV spreaders with its level-1 corresponding proteins in human PPIN (marked as green). C. Selection of spreaders in level-1 (level-1 spreaders are marked as yellow) and level-2 human proteins (level-2 spreaders are marked as green). Rest proteins in human PPIN are ignored to prevent overlap in the diagram.
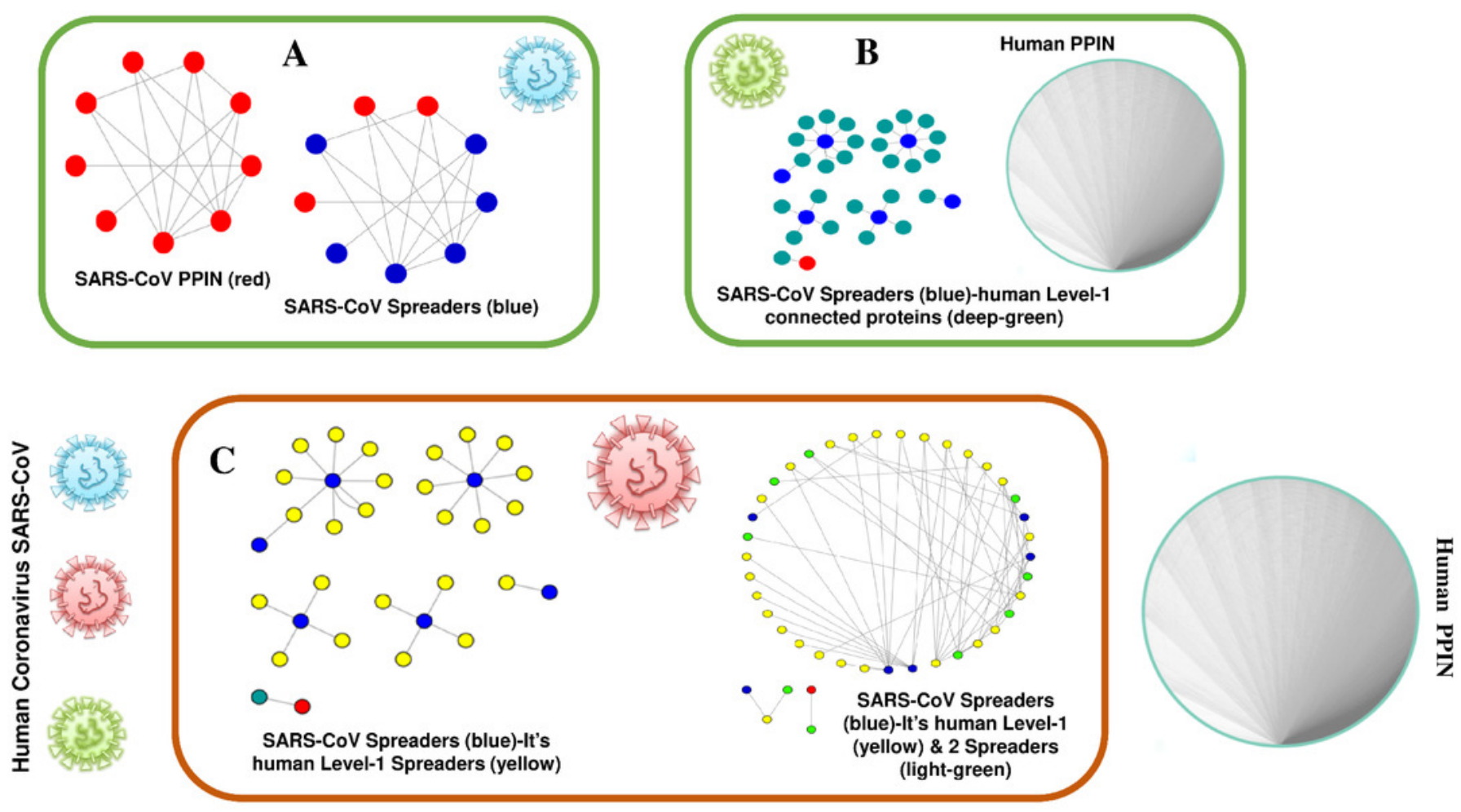
Figure 4

\section{SARS-COV PPIN.}

The PPIN consists of the interaction between SARS-CoV proteins only. It is a collection of 9 SARS-CoV proteins only (marked as red).

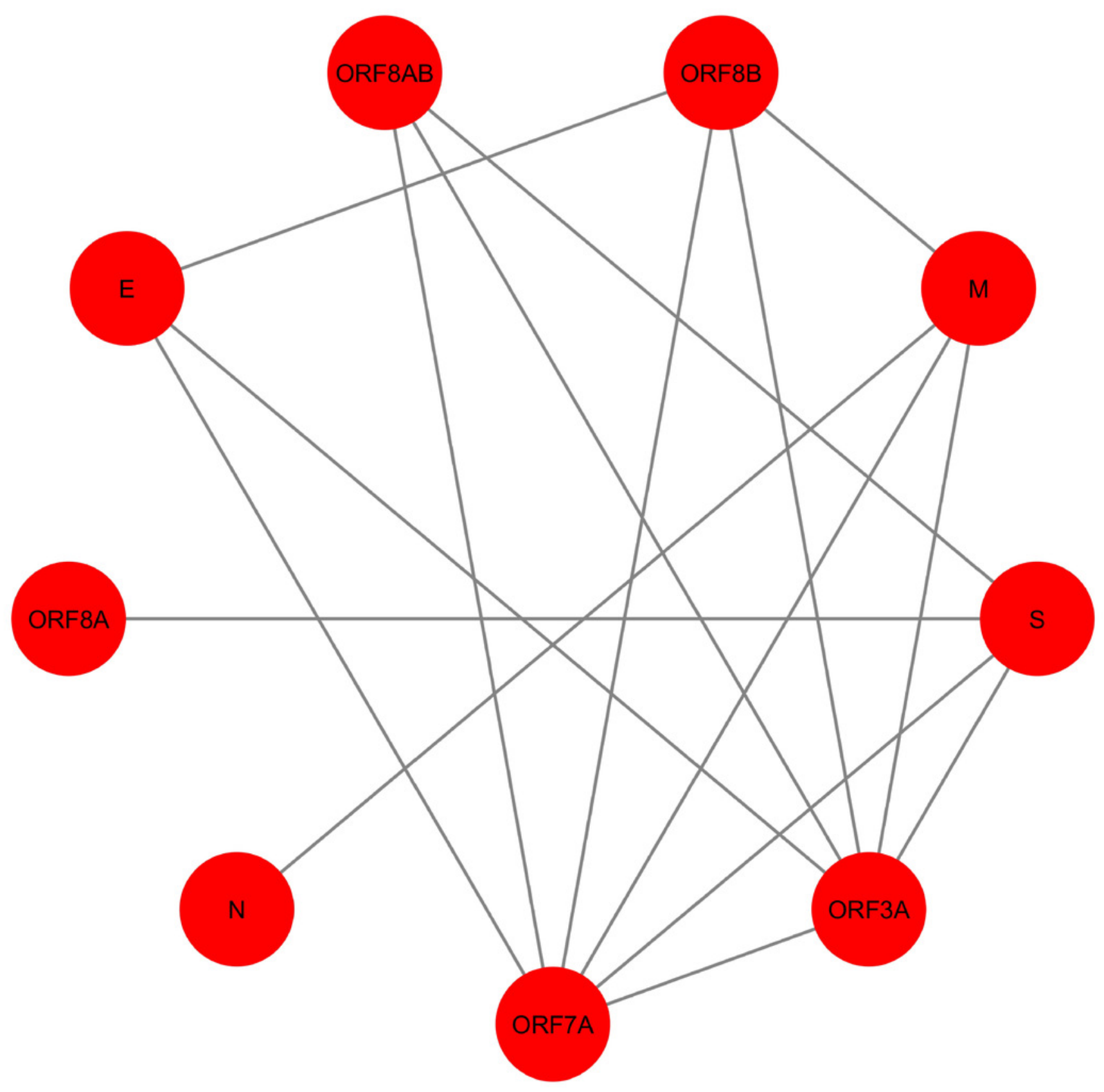


Figure 5

SARS-CoV-human PPIN (level-1).

The PPIN consists of the interaction between SARS-CoV and human proteins. The blue node represents SARS-CoV spreaders, while the yellow node represents SARS-CoV s level-1 human spreaders. The thickness of the edges varies with the order of ranking. 


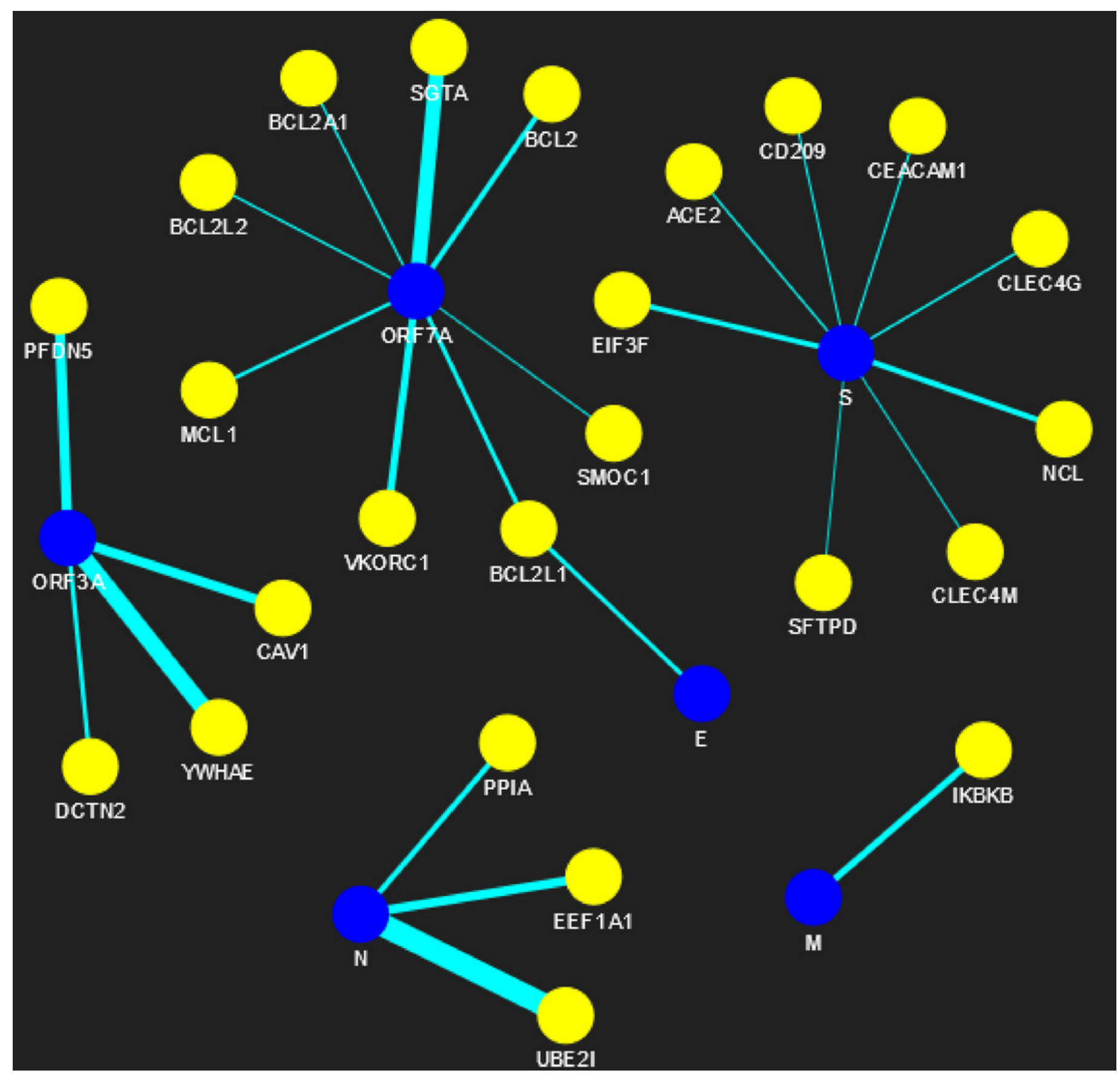


Figure 6

SARS-CoV-human PPIN (level-1 and level-2).

The PPIN consists of the interaction between SARS-CoV and human proteins. The blue node represents SARS-CoV spreaders, while the yellow and green nodes represent SARS-CoV S level-1 and level-2 human spreaders. The thickness of the edges varies with the order of ranking. 


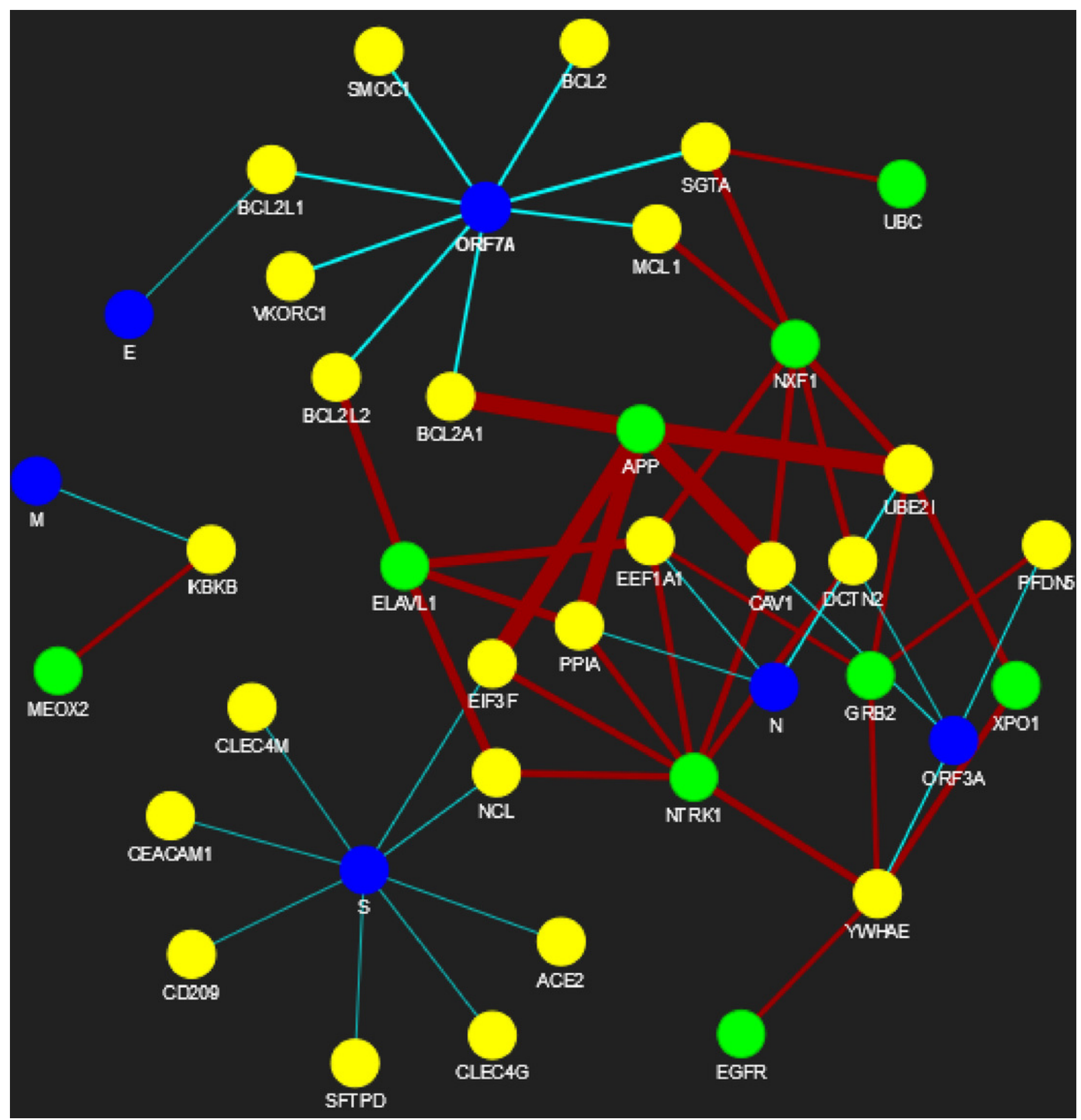

